\title{
Construction of a specialized control system for brush machine and rounding the cutting edges of a metal cutting tool
}

\author{
Petr Nikishechkin ${ }^{1, *}$, Nikita Grigoriev ${ }^{1}$, and Nadezhda Chervonnova ${ }^{1}$ \\ ${ }^{1}$ MSTU STANKIN, 127055 Vadkovskiy per. 3a, Moscow, Russia
}

\begin{abstract}
The basic aspects of preparing a cutting tool for applying wearresistant coatings to it, in particular, the use of brush technology to round its cutting edges, are investigated. A structural model for constructing a specialized brush machine control system has been developed and the basic aspects of its development have been determined.
\end{abstract}

\section{Research of brush technology for cutting tool edges}

To date, the task of preparing a metal-cutting tool for applying protective coatings to it is a difficult and urgent task, since the geometry of the tools is complicated, and the complexity of processing all the edges to give them the correct microgeometry and subsequent highquality coating is complicated. Similar problems are usually solved by developing specialized machines, often designed for a specific range of cutting tools from a particular manufacturer. Also, often these machines are controlled using complex control systems, which ultimately significantly increases their total cost.

In this paper, we consider the brush technology of cutting edge processing for a metal cutting tool, as well as aspects of constructing a control system for such an installation.

The principle of the brush technology is to remove the coating layer from the cutting edge of the tool with a special brush (Fig. 1).

\footnotetext{
* Corresponding author: pnikishechkin@gmail.com
} 


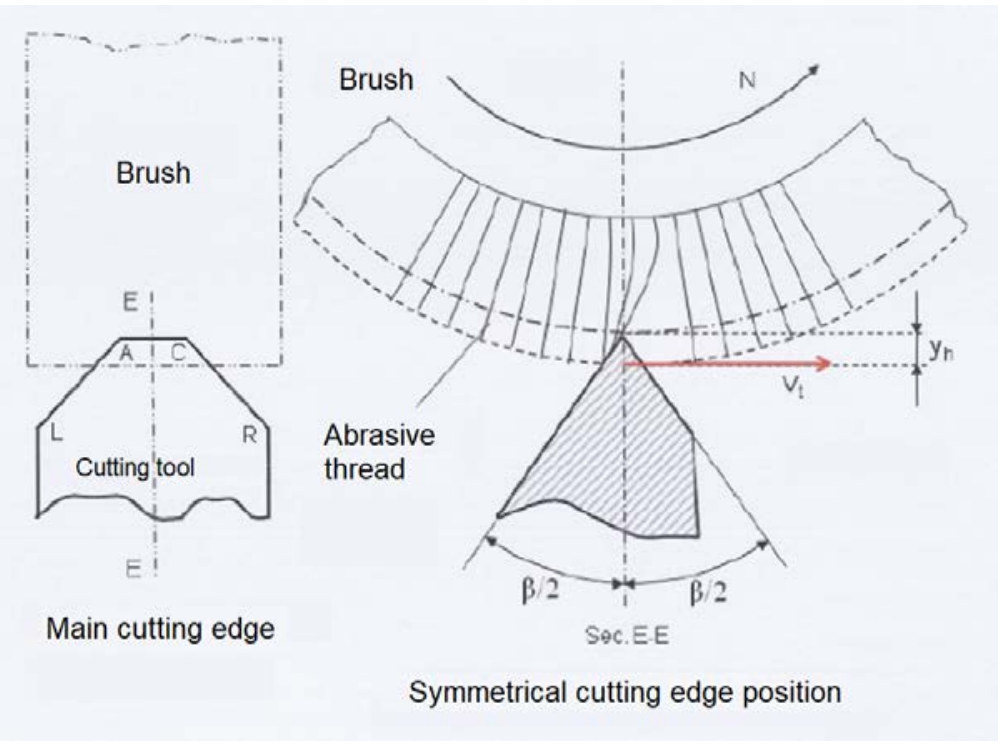

Fig. 1. Brush tool processing technology.

There are many factors that influence the choice of parameters for rounding the cutting edge of a tool, namely: type of processing, speed, feed, cutting depth, friction coefficient, material of a part, macrogeometry of a tool, alloy hardness, alloy composition, coating structure, coating thickness and other factors.

Depending on the type of processing, speed, feed, cutting depth, material of the part, macrogeometry of the tool and other factors, the degree of rounding of the cutting edge is selected.

In this research, it was found that the brush technology has many other advantages, namely: the possibility of separate processing of the tool surface, the creation of individual geometry for the tool surfaces, the constancy of the radius of rounding along the length of the tool, automation of the rounding process, the possibility of using different brushes for one technological the process, the possibility of rounding the edges on all instruments of almost any shape and the ease of mastering the technology.

\section{Development of a structural model for controlling a brushing machine}

During the research, a structural model was constructed that displays the main components of the brush machine and its control system, and their interaction with each other (Fig. 2). The model includes the following components: a control system consisting of a terminal and a computing core, peripheral devices for driving the axes of the complex, as well as the brush machine itself, for which a kinematic scheme was developed. 


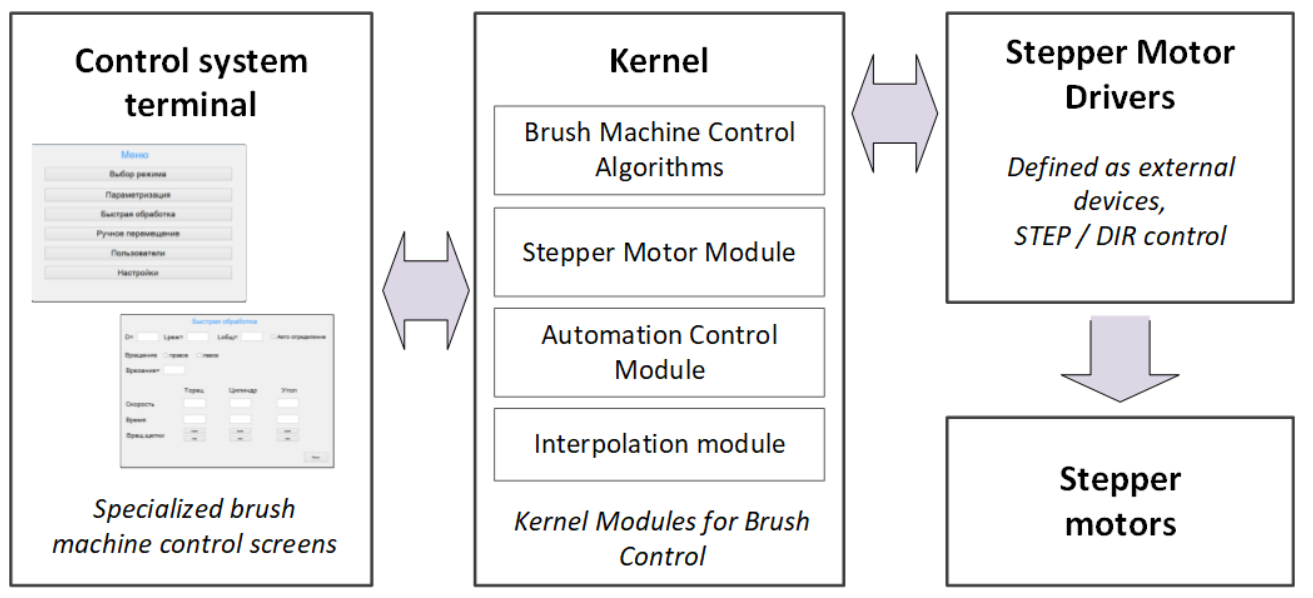

Fig. 2. Structural model of brush control.

The most important element of the complex is an adapted control system, which includes a computing core that performs all the computing and control operations, as well as a flexible operator interface that allows using process parameters to control the process for various types of tools and their dimensional characteristics.

At the same time, this control system must have enough functionality to control the 6 axes of the brushing machine, but without the redundant functionality found in CNC systems, such as frame previews, various interpolation algorithms, etc. It is assumed that the possibility of operating the control system on various hardware platforms, including domestic processors, should be provided for the possibility of use in closed tool factories with high security requirements.

The direct control object is a brush machine. The developed and presented kinematic scheme of the brushing machine reflects all the controlled axes of the complex, their type (circular / linear) and their construction. As can be seen from the figure, the brushing machine is a 6-axis equipment: XYZ axes are linear, ABC are angular (rotary) axes. Processing of the cutting edge on the brushing machine is carried out by moving a special brush along the cutting surfaces and removing a layer of excess material from them. Using 6 moving axes, the brushing machine covers a wide range of cutting tools.

\section{Development of a specialized brush machine control system}

One of the most important tasks is the creation of a control system adapted for the implementation of brush technology for tool processing in an automated mode. The development of this control system for the brushing machine is supposed to be carried out by adapting existing practices for the AxiOMA Control CNC system being developed at MSUT "STANKIN", in the creation of which I also took part. This CNC system is a domestic solution that allows you to solve the problems of controlling a wide range of machine equipment. The software part of the CNC system consists of two main components: the control system terminal, with which the operator interacts, and the core of the system, which implements all mathematical control algorithms (interpretation of the control program, interpolation, frame preview, etc.), as well as modules for work with external devices. Moreover, initially the control system is aimed at solving the problem of controlling high-precision machine equipment, and is not designed to solve specialized tasks, for example, controlling the developed brush machine. 
To adapt and refine the system for controlling the brushing machine, it is necessary to develop additional modules in the main components of the control system and carry out appropriate tests of their operation.

In the terminal part, it is necessary to create specialized screens for controlling the brushing machine, since the regular terminal screens of the CNC system have a different orientation and will not allow for the parameterization of the developed complex.

As for the control functions of the brushing machine, in contrast to the standard tasks solved by the CNC system, it is not necessary to achieve micrometric accuracy when controlling the axes to implement the brush technology of surface treatment of the cutting tool. Accordingly, the use of expensive high-precision servo drives is a redundant solution. In this regard, it was decided to use stepper motors to drive all the axes of the complex. At the same time, for their control it is necessary to develop modules for interacting with stepper motor drivers, as well as specialized algorithms for their control, which are absent in the standard CNC system.

Thus, in the core of the control system, a set of modules is developed for implementing specialized brush machine control algorithms, as well as modules for interacting with hardware devices - stepper motors that implement the movement of all axes of the brush machine, and an electro-automation control module for monitoring the processing process.

In the terminal part of the control system, several screens were developed to select the required operating mode of the complex and set the main parameters of the fast processing mode (Fig. 3). It is assumed that the terminal part will be implemented on the basis of a compact industrial terminal panel running Windows CE.

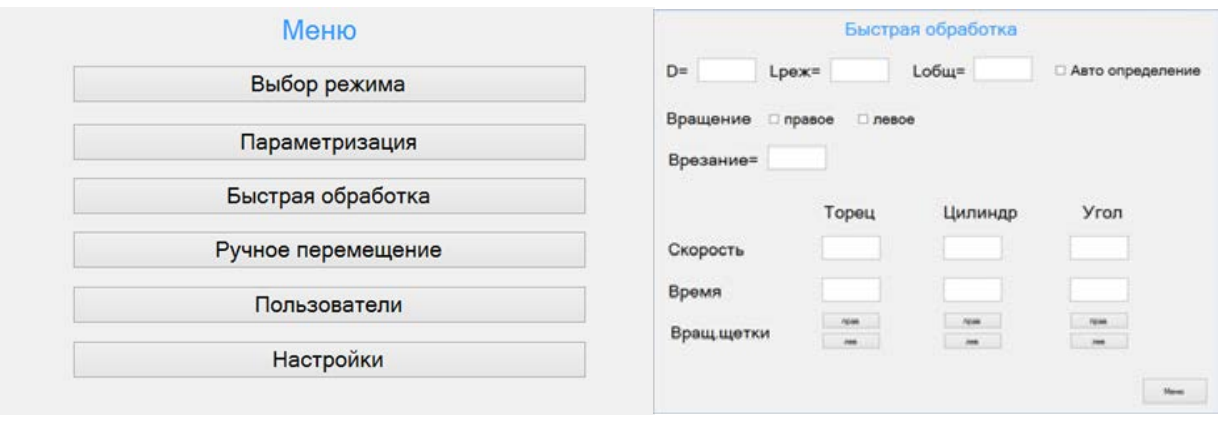

Fig. 3. Basic terminal screens for brush control.

In the kernel of the control system, modules for working with stepper motors and their control algorithms are currently being developed and debugged. Debugging is carried out on the basis of a specialized stand simulating the operation of a brush machine.

\section{Conclusion}

The hardware and software complex proposed for development will allow preparing the cutting tool for applying protective coatings to it by rounding its cutting edges in order to give microgeometry the necessary configuration and size. This will be implemented using a "brush" technology, while, unlike analogues using this technology, the complex will operate in an automated mode according to processing parameters predefined by operators.

A research was carried out on the possibilities of using brush technology for pretreatment of a cutting tool. A structural model has been developed that displays the main components of the brush machine and its control system, and their interaction with each other. The main tasks that automate the brush cutting tool processing technology are 
systematized, the basic algorithm of the complex's operation, as well as the basic modules for automating this process, are developed.

\section{References}

1. P. Nikishechkin, N. Chervonnova, A. Nikich, Approach to the construction of specialized portable terminals for monitoring and controlling technological equipment, In: MATEC Web Conf. Volume 224. International Conference on Modern Trends in Manufacturing Technologies and Equipment (ICMTMTE 2018), pp. 1-9 (2018)

2. N.S. Grigoriev, P.A. Nikishechkin, I.A. Kovalev, Research of the possibilities of using brush technology for rounding the cutting edges of a metal cutting tool, Collection of articles of the IV International scientific-practical conference "Modern science: current issues, achievements and innovations". Penza: ICSN "Science and Enlightenment", Part 1, pp. 82-84 (2018)

3. R. Nezhmetdinov, P. Nikishechkin, A. Nikich, Approach to the Construction of Logical Control Systems for Technological Equipment for the Implementation of Industry 4.0 Concept, In: 2018 International Russian Automation Conference (RusAutoCon). Sochi: IEEE, pp. 1-4 (2018)

4. P.A. Nikishechkin, N.S. Grigoriev, Construction of a specialized control system for rounding cutting edges on a metal cutting tool with the aim of high-quality application of protective coatings on it, Proceedings of the XVII international conference "Systems for design, technological preparation of production and management of the stages of the life cycle of an industrial product (CAD/CAM/PDM-2017)”, pp. 347-350 (2017)

5. P.A. Nikishechkin, N.Yu. Chervonnova, A.N. Nikich, Approach to Development of Specialized Terminals for Equipment Control on the Basis of Shared Memory Mechanism, In: Proceedings of the 4th International Conference on Industrial Engineering (ICIE-2018). (2018)

6. P.A. Nikishechkin, I.A. Kovalev, A.N. Nikich, An approach to building a crossplatform system for the collection and processing of diagnostic information about working technological equipment for industrial enterprises, MATEC Web Conf. Volume 129, International Conference on Modern Trends in Manufacturing Technologies and Equipment (ICMTMTE 2017). (2017)

7. G. Martinov, I. Kovalev, A. Al Khoury, Construction of a Specialized CNC System for Thread Grinding Machines, In: 2018 International Russian Automation Conference (RusAutoCon). Sochi: IEEE. (2018)

8. G.M. Martinov, P.A. Nikishechkin, A.S. Grigoriev, Organizing Interaction of Basic Components in the CNC System AxiOMA Control for Integrating New Technologies and Solutions,In: Automation and Remote Control, Vol. 80, No. 3, pp. 584-591 (2019)

9. S. Grigoriev, G. Martinov, An Approach to Creation of Terminal Clients in CNC System, In: 3rd Russian-Pacific Conference on Computer Technology and Applications. Vladivostok, pp. 1-4 (2018) 